\title{
A Note on Obstructions to Clustered Planarity
}

\author{
Jamie Sneddon \\ Department of Mathematics \\ The University of Auckland, Auckland, New Zealand \\ j.sneddon@auckland.ac.nz \\ Paul Bonnington \\ Monash e-Research Centre \\ Monash University, Melbourne, Australia \\ paul. bonnington@adm.monash.edu.au
}

Submitted: Apr 12, 2010; Accepted: Jul 27, 2011; Published: Aug 5, 2011

Mathematics Subject Classifications: 05C10, 05C20, 05C83

\begin{abstract}
A planar digraph $D$ is clustered planar if in some planar embedding of $D$ we have at each vertex the in-arcs occurring sequentially in the local rotation. By supplementing the operations used to form the usual minors in Kuratowski's theorem, clustered planar digraphs are characterised.
\end{abstract}

\section{Introduction}

Kuratowski's theorem is a well known result that characterises planar graphs. A (topological) minor of a graph $G$ is formed by some combination of three operations: vertex removal, edge removal and smoothing. Smoothing involves replacing two edges meeting at a degree two vertex with a single edge. Under the implied ordering the minimal non-planar graphs, or so-called obstructions to planarity, are $K_{3,3}$ and $K_{5}$.

Kuratowski's theorem and Robertson and Seymour's epic "fundamental theorem" for topological graph theory (culminating in [5]) do not apply to digraphs. When restrictions are placed on the nature of the digraph embedding, the operations used to form digraph minors may be restricted or modified; it is no longer certain that the set of obstructions under a restricted or modified partial order is finite. Bonnington et. al. [1, 2] have investigated Eulerian planar digraphs; that is, digraphs with planar embeddings in which the in-arcs and out-arcs alternate around each vertex. Pisanski [4] has investigated the genus distribution and embeddings of graphs with clustered in-arcs at each vertex, and clustered planar graphs in particular. This paper investigates extending the use of minors 
(in an altered sense) for digraphs with conditions placed on the nature of the directed embedding to determine obstructions to a digraph property.

A vertex $x$ in a digraph is called a source (respectively sink) if all of the arcs at $x$ are out-arcs (in-arcs). An ss-digraph is a digraph in which every vertex is either a sink or a source. Clearly, the underlying graph of an ss-digraph is bipartite.

A digraph $D$ is clustered planar if it has a planar embedding in which at each vertex the in-arcs occur sequentially in the local rotation. In this paper we provide a Kuratowski-type theorem for clustered planarity. As an intermediary step, we will classify the ss-digraphs which are clustered planar, and show how this relates to planar bipartite graphs. Of use in classifying clustered planar digraphs is the property of planarity of ss-digraphs; such digraphs are said to be ss-planar, having the property of ss-planarity.

A source-path between two vertices $x$ and $y$ consists of two $\operatorname{arcs}(w, x)$ and $(w, y)$ for some vertex $w$ of degree two, and is denoted $\stackrel{\leftrightarrow}{x y}$. Similarly, a sink-path between $x$ and $y$ consists of $\operatorname{arcs}(x, w)$ and $(y, w)$ where $w$ has degree two, and is denoted $\vec{x} y$. Collectively we call these s-paths. A digraph $H$ is an s-path subdivision of a graph $G$ if it has the vertices of $G$ and an arc or an s-path in $H$ for each edge in $G$. Note that the underlying graph of $H$ is a subdivision of $G$.

Four operations preserve ss-planarity:

1. Vertex removal.

2. Arc removal (the directed equivalent of edge removal).

3. The double smoothing operation is only applied to adjacent degree two vertices. If there exist vertices $w, x, y$ and $z$ with $\operatorname{arcs}(w, x),(y, x)$ and $(y, z)$, where $x$ and $y$ are degree two vertices, then the double smoothing operation removes these arcs and the vertices $x$ and $y$, and introduces the new $\operatorname{arc}(w, z)$.

4. The cut inversion operation has the effect of reversing the direction of all of the arcs at a vertex $x$. Each sink-path $\underset{x y}{x}$ is replaced by the arc $(y, x)$. For each remaining $\operatorname{arc}(x, z)$ at $x$ (which is not on a sink-path), a new vertex $\hat{z}$ is introduced, and the arc $(x, z)$ is replaced by the source-path $\stackrel{\leftrightarrow}{x z}$ through $\hat{z}$. Each source-path $\stackrel{\leftrightarrow}{x y}$ is replaced by the arc $(x, y)$, and for each remaining arc $(z, x)$ at $x$ (which is not on a source-path), a new vertex $\hat{z}$ is introduced, and the $\operatorname{arc}(z, x)$ is replaced by $\vec{x} z$ through $\hat{z}$. Cut inversions are only performed when the resulting digraph is smaller, in the sense outlined below. Figure 1 illustrates a cut inversion operation.

An operation may be performed on a digraph $H=(V, A)$ if the operation reduces the number of vertices and arcs, $M_{1}=|V|+|A|$. Additionally, an operation may be performed if this measure $M_{1}$ remains constant, and the measure $M_{2}=$ (\# sources) - (\# sinks) is reduced. An example of this kind of minor forming is shown in Figure 1. In this way, the standard partial order of topological minors is modified.

Any double smoothing, vertex removal or arc removal reduces $M_{1}$. A cut inversion may be performed at a vertex $x$ of degree $n>2$ if there are at least $\lceil(n+1) / 2\rceil$ s-paths 


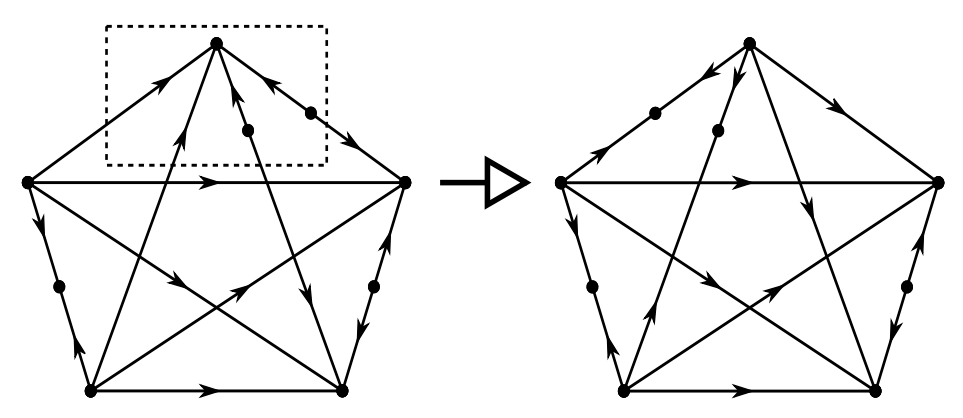

Figure 1: The cut inversion operation forming $J_{3}$ (which is $\Sigma_{2}$ ) as an ss-minor of $J_{2}$. The cut inversion is shown as a dashed box.

at $x$, or if there are exactly $n / 2$ source-paths at $x$. A cut inversion either reduces the number of s-paths in a digraph, or keeps this number constant but increases the number of sinks.

These operations and measures form a partial order of ss-minors in which it turns out there are two obstructions to ss-planarity. The first, $\Sigma_{1}$, is formed in a straightforward manner from $K_{3,3}$ by directing all edges of $K_{3,3}$ from one of the sets of the bipartition to the other. The second more interesting obstruction, $\Sigma_{2}$, is built from $K_{5}$ as an s-path subdivision by labelling two of the vertices as sinks and three as sources. Edges are replaced by arcs from sources to sinks, sink-paths between sources, and a source-path between the sinks. The underlying graph of $\Sigma_{2}$ is called $K_{5}^{b}$. This second obstruction is shown on the right in Figure 1.

\section{Obstructions to ss-planarity}

Theorem 1 Suppose $H$ is an obstruction to ss-planarity. Then $H$ is either $\Sigma_{1}$ or $\Sigma_{2}$.

Proof. Since the underlying graph $G$ of $H$ is non-planar, it must contain (by Kuratowski's theorem [3]) a subdivision of either $K_{3,3}$ or $K_{5}$. An ss-minor can be formed from $H$ in the same way, using the same operations of vertex and arc removals on $H$ as are performed on $G$.

Moreover, the subdivided edges of the corresponding Kuratowski subgraph of $H$ can be double smoothed to either arcs or s-paths. Let $J$ be the s-path subdivision of $K_{3,3}$ or $K_{5}$ obtained in this way.

Suppose $J$ is an s-path subdivision of $K_{3,3}$. Let $X$ and $Y$ be the two sets of vertices in the bipartition of $K_{3,3}$, let $i$ be the number of sources in $X$, and let $k$ be the number of sources in $Y$. Without loss of generality, we suppose $i \geq k$, and denote such an s-path subdivision of $K_{3,3}$ with the notation $J_{i, k}$. There are ten such non-isomorphic s-path subdivisions of $K_{3,3}$. Cut inversions operations reduce all s-path subdivisions of $K_{3,3}$ to $J_{3,0}$, as the following sequences of cut inversions show.

$$
J_{3,3} \rightarrow J_{3,2} \rightarrow J_{2,2} \rightarrow J_{2,1} \rightarrow J_{2,0} \rightarrow J_{3,0}
$$



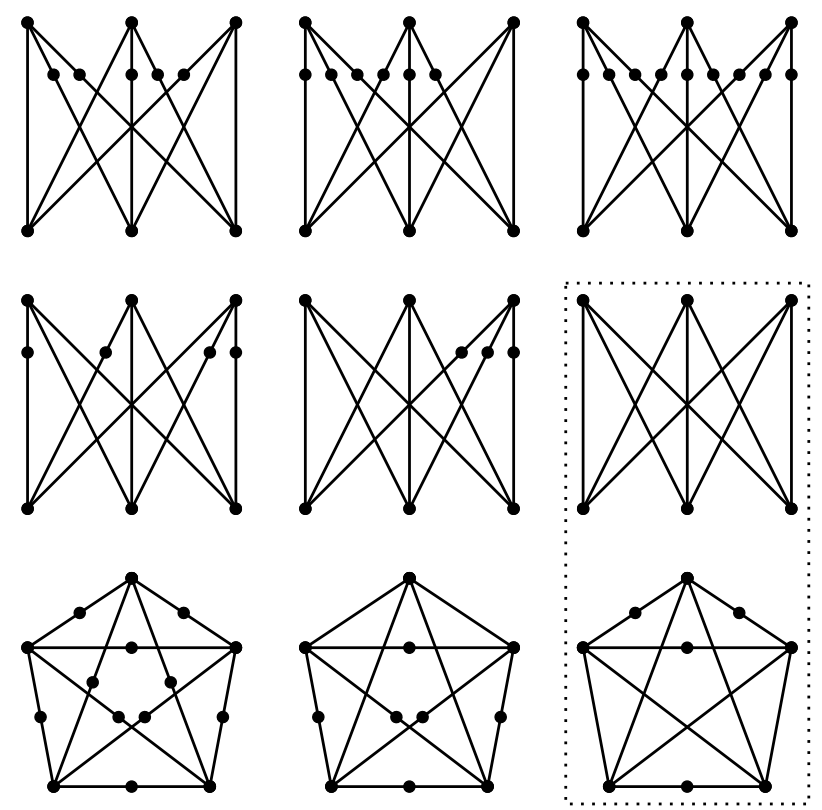

Figure 2: The nine obstructions to bipartite planarity without cut inversion allowed. The obstructions with cut inversion, $K_{3,3}$ and $K_{5}^{b}$, are in the lower right.

$$
J_{0,0} \rightarrow J_{1,0} \rightarrow J_{1,1} \rightarrow J_{2,1} \rightarrow J_{3,1} \rightarrow J_{3,0}
$$

It follows that if $G$ contains a subdivision of $K_{3,3}$, then $H$ has $J_{3,0}=\Sigma_{1}$ as an ss-minor.

Suppose $J$ is an s-path subdivision of $K_{5}$. In this case, the degree 4 vertices are partitioned into two sets $X$ and $Y$ of sources and sinks respectively. Vertices in the same set have an s-path between them; vertices in different sets have an arc from the vertex of $X$ to the vertex of $Y$. There are six such non-isomorphic s-path subdivisions of $K_{5}$, which we label $J_{|X|}$. With cut inversion operations, we find $J_{0} \rightarrow J_{1} \rightarrow J_{2} \rightarrow J_{3}$ (the final operation keeping $M_{1}$ constant while reducing $M_{2}$ as shown in Figure 1) and $J_{5} \rightarrow J_{4} \rightarrow J_{3}$. It follows that if $G$ contains a subdivision of $K_{5}$, then $H$ has $J_{3}=\Sigma_{2}$ as an ss-minor.

Without the cut inversion operation there are 16 obstructions to ss-planarity, corresponding to the different forms of $J_{i, k}$ and $J_{|X|}$ in the proof. Without the measure $M_{2}$ there are three obstructions to ss-planarity, as the cut inversion $J_{2} \rightarrow J_{3}$ would not be possible.

These operations preserving ss-planarity of digraphs can be used as undirected operations which preserve bipartite planarity of graphs. The corresponding underlying graphs of $\Sigma_{1}$ and $\Sigma_{2}$ are the obstructions to planarity of bipartite graphs. Without cut inversion there are nine obstructions to bipartite planarity, corresponding to the non-isomorphic underlying graphs of the digraphs $J_{i, k}$ and $J_{|X|}$ in the proof; these are shown in Figure 2.

Corollary 2 The obstructions to bipartite planarity (under our implied ordering which preserves bipartiteness) are $K_{3,3}$ and $K_{5}^{b}$. 


\section{Obstructions to Clustered Planarity}

We use Theorem 1 to find the obstructions to clustered planarity. Note that all ss-planar digraphs are also clustered planar. We introduce a new operation which can be used successively to form a minor of any clustered planar digraph which is ss-planar.

The new operation for clustered planarity is an expansion operation. An expansion operation may be performed at any vertex $x$ which is neither a sink or a source. The expansion operation separates the in-arcs and the out-arcs at the vertex, forming two new vertices. One of the new vertices is a sink, and other other is a source. The vertex $x$ is removed and replaced by the vertices $x^{+}$and $x^{-}$and the $\operatorname{arc}\left(x^{+}, x^{-}\right)$. Every arc $(x, y)$ is replaced by the $\operatorname{arc}\left(x^{+}, y\right)$, and every $\operatorname{arc}(z, x)$ is replaced by the $\operatorname{arc}\left(z, x^{-}\right)$. (The vertices $x^{+}$and $x^{-}$are a source and sink respectively). This new operation preserves clustered planarity.

This operation increases the value of $M_{1}$ by two. The resulting minor is bigger than the original digraph (in that it has one more edge and one more vertex). Accordingly, we introduce a new (and more important) measure $M_{0}$. Simply, let $M_{0}$ be the number of vertices which have both in-arcs and out-arcs.

Given a digraph $D$, a digraph formed by one of the operations discussed above (expansion, cut inversion, double smoothing, arc removal and vertex removal) is a minor in the extended partial order if the measure $M_{0}$ is reduced, or $M_{0}$ remains constant and $M_{1}$ is reduced, or if $M_{0}$ and $M_{1}$ remain constant and $M_{2}$ is reduced.

Lemma 3 Obstructions to clustered planarity (under the extended partial order) are ssdigraphs.

Proof. Suppose $D$ is an obstruction to clustered planarity that is not an ss-digraph; that is, $M_{0}>0$. Then it has a vertex $x$ which is neither a sink or a source, and expanding that vertex forms a minor of $D$ which is not clustered planar, a contradiction of the minimality of the supposed obstruction $D$. Hence all obstructions have $M_{0}=0$.

Theorem 4 The obstructions to clustered planarity under the extended partial order are $\Sigma_{1}$ and $\Sigma_{2}$.

Proof. The proof follows immediately from Lemma 3 and the proof of Theorem 1.

The set of obstructions depends on the operations (and measures) used. Other partial orders using different operations and measures result in different sets of obstructions. It is possible to use only operations which do not increase the number of vertices or arcs when forming a minor; however the arguments required are longer and more complicated, with eleven operations, four measures and resulting in ten obstructions to clustered planarity [6]. 


\section{References}

[1] C.P. Bonnington, M. Conder, P. McKenna and M. Morton, Embedding digraphs on orientable surfaces, J. Combin. Theory Ser. B, 85, (2002), no. 1, 1-20.

[2] C.P. Bonnington, N. Hartsfield and J. Širán̆, Obstructions to directed embeddings of Eulerian digraphs in the plane, European J. Combin., 25 (2004), no. 6, 877-891.

[3] K. Kuratowski, Sur le problème des courbes gauches en topologie, Fund. Math. 15, (1930), 271-283.

[4] Pisanski, T., private communication, 2009.

[5] Robertson, S. and Seymour, P., Graph Minors. XX. Wagner's Conjecture, J. Combin. Theory Ser. B, 92, (2004), no. 2, 325-357.

[6] J. Sneddon, Minors and embeddings of digraphs, PhD thesis, The University of Auckland, (2004). 